\title{
Surface Heterogeneities Matter in Fast Scan Cyclic Voltammetry Investigations of Catecholamines in Brain with Carbon Microelectrodes of High-Aspect Ratio: Dopamine Oxidation at Conical Carbon Microelectrodes
}

\author{
Alexander Oleinick, ${ }^{1}$ Isabel Álvarez-Martos, ${ }^{2}$ Irina Svir, ${ }^{1, z}$ Elena E. Ferapontova, $\overbrace{}^{2, z}$ \\ and Christian Amatore $\mathbb{B} 1,3, *, \mathrm{z}$ \\ ${ }^{I}$ PASTEUR, Département de chimie, École normale supérieure, PSL University, Sorbonne Université, CNRS, 75005 \\ Paris, France \\ ${ }^{2}$ Interdisciplinary Nanoscience Center (iNANO), Aarhus University, DK-8000 Aarhus C, Denmark \\ ${ }^{3}$ State Key Laboratory of Physical Chemistry of Solid Surfaces, College of Chemistry and Chemical Engineering, \\ Xiamen University, Xiamen 361005, People's Republic of China
}

\begin{abstract}
Fast Scan Cyclic Voltammetry (FSCV) at high aspect ratio carbon microelectrodes shows adequate high temporal and spatial resolution for in vivo analysis of catecholamines. Though the presence of their surface heterogeneities has been recognized since their earliest introduction for in vivo measurements in the brain, the kinetic consequences on the measurements have not been investigated and FSCV measurements are treated based on pre- and post-calibrations. We establish here that surface heterogeneities play a consequent dynamic role on the oxidation of dopamine taken as an example of catecholamines. Hence, the FSCV current peak intensities do not scale with the scan rate $v$ or its square root. This is rationalized with a simple model involving a co-existence of at least two types of surface nanodomains with different electrochemical reactivities and different time responses. At low scan rates $\left(<100 \mathrm{~V} \mathrm{~s}^{-1}\right)$ dopamine molecules that initially adsorbed onto non-electroactive nanodomains have enough time to migrate toward highly electroactive ones so all molecules initially adsorbed on the whole electrode surface may be oxidized during one FSCV cycle. Current peak intensities then increase proportionally to the scan rate. However, above $100 \mathrm{~V} \mathrm{~s}^{-1}$, dopamine migration between sites starts to be kinetically limited so that FSCV current peak intensities do not increase any more proportionally to the scan rate. Ultimately, i.e., above $1000 \mathrm{~V} \mathrm{~s}^{-1}$, the dopamine exchange between sites is almost totally blocked so only dopamine molecules initially adsorbed on the electroactive surface nanodomains may be oxidized; the current peak intensities then increase again proportionally with the scan rate though with a smaller slope than that observed at small scan rates. Since carbon fibers with large aspect ratios are frequently used in brain investigations, this effect should be a concern when extracting quantitative results even when each carbon fiber response is properly pre- or post-calibrated using the exact CV waveform and scan rate used during the in vivo measurements.

(C) The Author(s) 2018. Published by ECS. This is an open access article distributed under the terms of the Creative Commons Attribution 4.0 License (CC BY, http://creativecommons.org/licenses/by/4.0/), which permits unrestricted reuse of the work in any medium, provided the original work is properly cited. [DOI: $10.1149 / 2.0071812 \mathrm{jes}$ ]
\end{abstract}

Manuscript submitted April 25, 2018; revised manuscript received June 13, 2018. Published July 18, 2018. This paper is part of the JES Focus Issue on the Brain and Electrochemistry Honoring R. Mark Wightman and Christian Amatore.

Release and modulation of neurotransmitters fluxes and concentrations are essential in complex organisms since they regulate many key functions by transferring information between cells such as neurons in the brain, neurons and muscle cells as well as through stimulating specific actions in endocrine-related systems. Albeit their unique and ubiquitous functions are well recognized today, the very existence and identification of neurotransmitters has long been a matter of debate up to the end of the second third of the past century, ${ }^{1-3}$ and still needs to be better understood. This explains why characterizing fluxes and nature of neurotransmitters release is still stimulating a quest for analytical methods sufficiently accurate, specific and sensitive to investigate dynamic effluxes and flows of neurotransmitters in vivo (e.g., within a living brain $)^{4-6}$ or ex vivo (e.g., at the single endocrine cell level, ${ }^{7-9}$ or intrasynaptically ${ }^{10-12}$ ) with an adequate temporal resolution. In this respect, electrochemical oxidation of neurotransmitters at carbon-based micro- and nanoelectrodes has established itself as the method of choice. This obviously stems from their excellent properties in terms of analyte specific and sensitive responses and sub-millisecond time resolution but also from their cheap, easy and versatile manufacturing. For example, carbon-disks made through exposing cross-sections of sealed carbon fibers have been used to monitor catecholamines transient release episodes in brain ${ }^{13-16}$ and then adapted to characterize single vesicular exocytotic events at single cells levels through the "artificial synapse" strategy. ${ }^{7-12}$ Furthermore, carbon-disk microelectrodes responses to catecholamines oxidation have been extensively characterized under steady state conditions or through transient

*Electrochemical Society Fellow.

${ }^{\text {z}}$ E-mail: christian.amatore@ens.fr; elena.ferapontova@inano.au.dk; irina.svir@ens.fr voltammetry over a wide range of voltammetric scan rates and proved superior to metallic ones. ${ }^{17,18}$

Carbon-based electrodes have important molecular surface properties that are essential for time-resolved monitoring of catecholamine release and concentration modulation in the brain by fast-scan cyclic voltammetry (FSCV) $)^{13}$ or short-pulse chronoamperometry. ${ }^{14,15}$ Indeed, in the corresponding short time ranges of interest, the voltammetric waves remain overall bielectronic ${ }^{19}$ and controlled by adsorption (Langmuir isotherm) without significant interference of mass transport. ${ }^{18}$ This is essential to afford current peak intensities that are proportional to the scan rate $v$ rather than to $v^{1 / 2}$ as would occur for diffusion controlled oxidation waves. Finally, carbon-disks obtained through cross-section of etched carbon fibers behave as if their surfaces were homogeneously electroactive with respect to catechols and quinones electrochemistry as confirmed through chronoamperometry. ${ }^{20}$

However, this does not seem to be easily generalizable to other types of carbon microelectrodes achieved by different procedures..$^{21,22}$ In particular, it is noted that in many recent investigations aimed to monitor quantitatively catecholamine dynamic concentrations by FSCV in living brains, one relies more frequently onto cylindrical ${ }^{23-26}$ or pseudo-cylindrical/conical ${ }^{27}$ carbon fibers electrodes whose exposed shafts lengths greatly exceed their diameters (e.g., typically from $50 \mu \mathrm{m}$ to one - or, possibly, a few - hundred micrometers unsealed shafts vs. 5-7 $\mu \mathrm{m}$ radii). Such high-aspect ratios are generally favored since they allow overcoming the micrometric-scale heterogeneity of synapses distributions in brain areas of interest. ${ }^{23}$ However, in contrast with the near-homogeneity of electroactive properties of their cross-sections, ${ }^{17,18}$ carbon fiber shafts are known 
from their earliest use to display finely interwoven surface states with different electrochemical properties. ${ }^{28,29}$ Though usually dismissed during quantitative determination of catecholamines concentrations and fluxes by FSCV, such surface heterogeneities have certainly high analytical consequences due to differences in adsorption characteristics and interfacial electron transfer rate constants. ${ }^{30}$ Indeed, electron transfer rates ${ }^{31-35}$ as well as kinetic and thermodynamic parameters of catecholamines adsorption are expected to drastically depend on the exact molecular and electronic states of carbon surfaces.

Hence, quantitative analyses of the responses of cylindrical electrodes used in FSCV require pre- and post-calibrations in order to deduce instant neurotransmitters concentrations from current peak intensities measured from background subtracted voltammograms in the living brain. ${ }^{36}$ However, the validity of such calibrations implicitly assumes that the biological sites releasing the neurotransmitters are uniformly distributed within the probed tissue area as occurs in the calibrating solutions, which is generally not the case. ${ }^{37}$ In other words, this amounts to consider that all the electroactive surface areas distributed along the electrode shaft are accessible in identical ways during the in vivo measurement and during the pre- or post-calibrations. Would that be not the case, the values of the preand post-calibrated factors determined in solution for a specified scan rate may not apply when the electrode is placed in the brain. Indeed, diffusion of neurotransmitters between the release locations in the tissue and the adsorption sites at the electrode ${ }^{14,15}$ as well as between non-electroactive and electroactive sites distributed along the electrode shaft necessarily involve two additional time-scales that are absent during the calibrations.

Owing to the importance of cylindrical or pseudo-cylindrical/ conical carbon fibers electrodes for neurobiological investigations by FSCV we decided to examine if their surface electrochemical characteristics could kinetically impact the outcome of FSCV measurements. For this purpose, we decided to resort to the use of commercial conical carbon fiber electrodes in order to reduce variabilities related to in-house hand-made ones. Owing to its importance in brain studies, dopamine oxidation was chosen as a typical catecholamine substrate. Electrochemical waves were obtained and quantitatively analyzed based on fast scan cyclic voltammograms after classical background subtraction. ${ }^{38}$ This evidenced non-monotonous variations of current peaks intensities with the scan rate. Similarly, the descending branches of $\mathrm{CV}$ did not followed the anticipated characteristic adsorptive behavior $^{39}$ as occurs at carbon disks microelectrodes. ${ }^{17,18}$ These unexpected observations could not be explained through a competitive involvement of solution diffusion or electron-hopping in adsorbed films. Conversely, they could be qualitatively rationalized through considering that dopamine adsorbs onto electroactive and non-electroactive nanodomains interwoven over the electrode surface.

\section{Experimental}

Materials and reagents.-Dopamine and components of the buffer solution $\left(\mathrm{NaCl}, \mathrm{Na}_{2} \mathrm{HPO}_{4}\right.$ and $\left.\mathrm{NaH}_{2} \mathrm{PO}_{4}\right)$ were purchased from Sigma-Aldrich (Germany) and used as received. Ultrapure water (18 M $\Omega$, a Milli-Q $\mathrm{A}^{+}$water purification system, Millipore, Bedford, MA, USA) was used throughout the work. Stock solutions of dopamine were daily prepared in a $20 \mathrm{mM}$ phosphate buffer solution (PBS, pH 7.4) containing $0.15 \mathrm{M} \mathrm{NaCl}$ and protected from light. This work was primarily aimed at kinetic studies. For this reason we used rather large concentrations $(30 \mu \mathrm{M})$ of dopamine in order to reduce any errors associated with the measurement of faradaic current intensities by minimizing the relative contribution of the background currents. However, since the oxidation processes investigated are by definition concentration independent, ${ }^{19}$ this has no consequence on the kinetic results presented hereafter.

Electrochemical measurements.-Fast scan cyclic voltammograms (FSCV) were recorded in a three-electrode glass cell $(10 \mathrm{~mL}$ working volume) placed inside a Faraday cage and connected to an AUTOLAB PGSTAT 302N potentiostat equipped with an
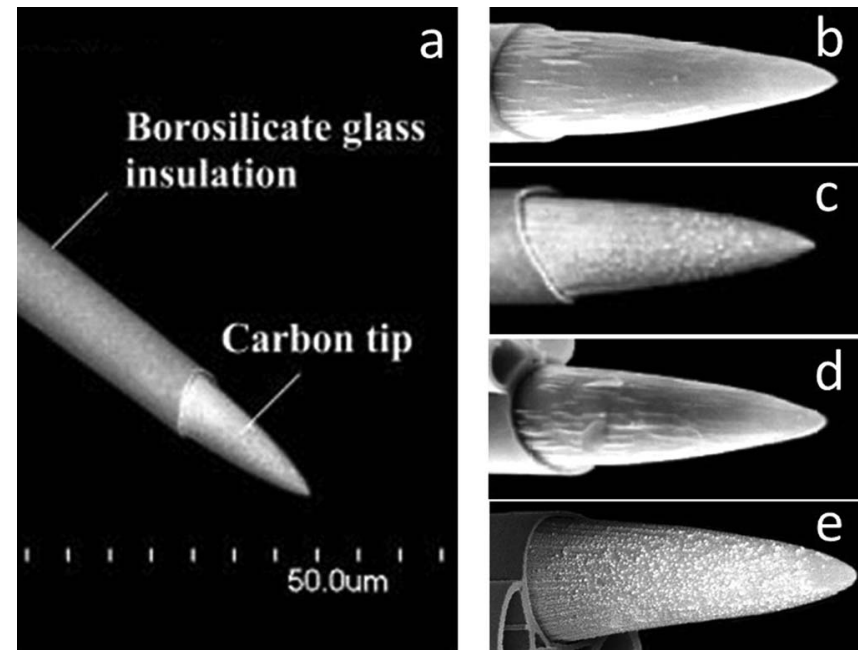

Figure 1. (a) SEM photograph of the Carbostar commercial conical carbonfiber electrodes similar to those used in this study according to the manufacturer; (b-e) Close views of four different conical tips as adapted from pictures displayed by Denes Budai/Kation Scientific Company on its web site and reproduced with permission (see also Reference 27 ).

ultra-fast sampling module and an impedance module (EcoChemie B.V., Utrecht, the Netherlands) and NOVA 1.10 software.

A pseudo reference electrode consisting of a $1.5 \mathrm{~mm}$ diameter $\mathrm{AgCl}$-covered $\mathrm{Ag}$ wire $(70 \mathrm{mV}$ vs. $\mathrm{Ag} / \mathrm{AgCl}$ in $3 \mathrm{M} \mathrm{KCl})$ and a platinum wire counter electrode were used. The working electrodes consisted of a series of commercial conical carbon Carbostar microelectrodes. According to the Kation Scientific Company specifications, ${ }^{27}$ their electrochemically active surface consists of $7 \mu \mathrm{m}$ diameter polyacrynitrile (PAN)-type graphite monofilament etched into a conical form and sealed in borosilicate glass (see Figure 1a). PAN-type carbon fibers contain carbon atom sheets that are haphazardly folded, or crumpled. That provides the carbon fibers with a high tensile strength perfectly suited to allow their tip to be etched into conical shapes ideal for penetrating in a living tissue without significant damage. However, this necessarily leads to morphological inhomogeneities of the exposed conical carbon surfaces as is apparent in the SEM micrographs in Figures 1b-1e. Voltammetric measurements obtained from each Carbostar electrode were duplicated or triplicated in order to ensure their reproducibility. Preliminary voltammetric investigations were performed with one type of Carbostar commercial conical carbon microelectrode noted CCFO (Kation scientific, USA), while the extended quantitative analyses were performed using a set of 4 similar Carbostar ones noted CCF1-4 (26 $\pm 4 \mu \mathrm{m}$ exposed length, $7 \mu \mathrm{m}$ diameter, E1011) from a single batch ordered from the same company (Kation scientific, USA) in order to allow a quantitative comparison of their behavior and assess experimental variabilities within a related batch of CCF microelectrodes.

The solution ohmic resistance was determined by two ways: (i) through electrochemical impedance spectroscopy at $100 \mathrm{kHz}$, thus providing a value of $27 \pm 2 \mathrm{k} \Omega$ upon assuming a simple RC equivalent circuit, or (ii) theoretically, based on the initial slope of the capacitive currents. ${ }^{40}$ The theoretical method indicated that resistances were ca. 2.5 times larger than the one deduced based on simple RC analyses, a fact that we consider to stem from the non-constant value of the capacitance along one CV (see below Figure 2) as well as from the presence of heterogeneities in the distribution of electroactive domains on the electrode surface. ${ }^{41,42}$

\section{Results}

Figure 2 shows a series of typical voltammograms recorded over the range of scan rates explored in this study at the Carbostar conical carbon fiber CCF0 microelectrode in PBS at $\mathrm{pH} 7.4$ without or with $30 \mu \mathrm{M}$ dopamine added. Qualitatively identical results were obtained 

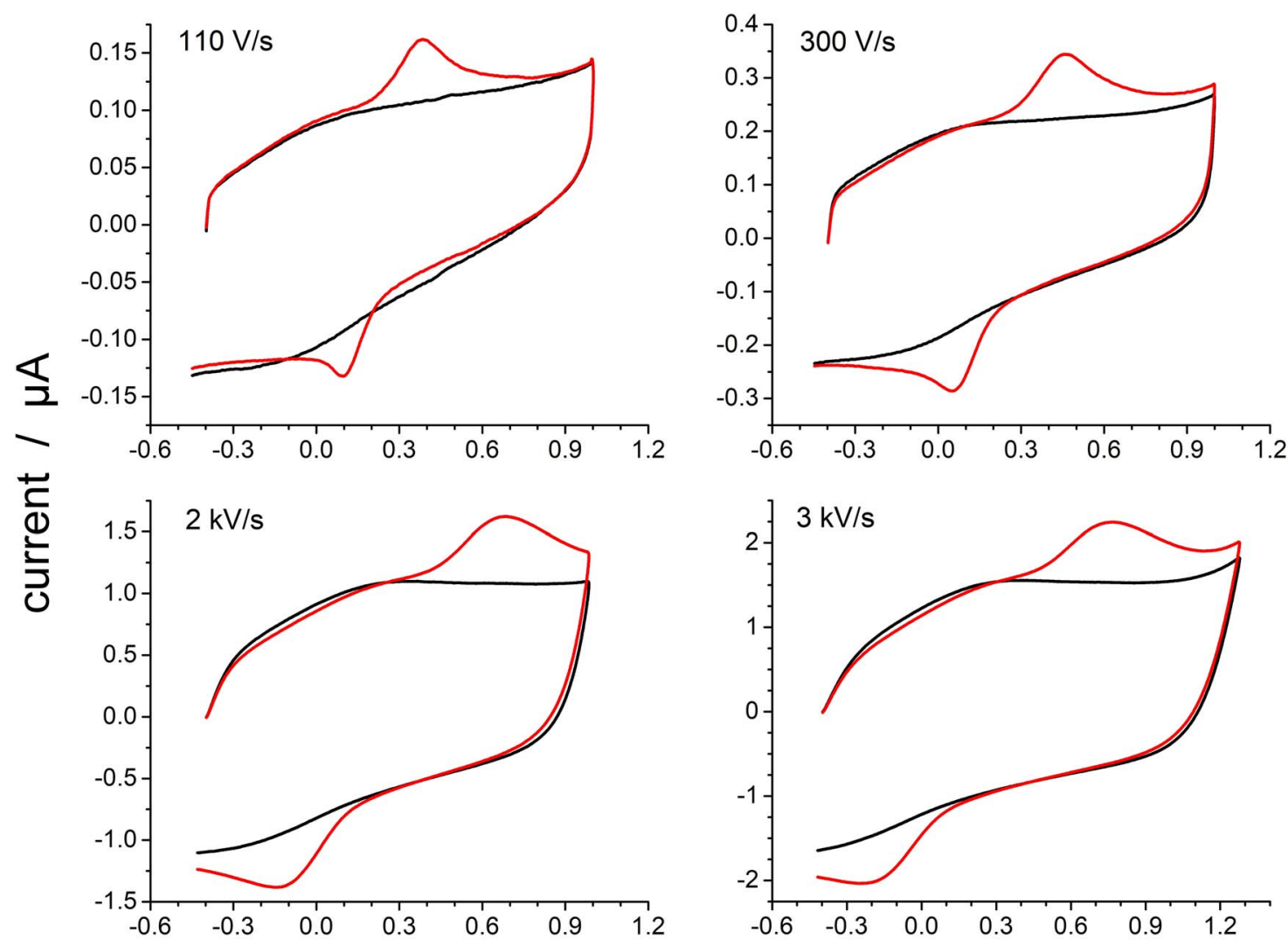

\section{$E / \mathrm{V}$ vs. $\mathrm{Ag} / \mathrm{AgCl}$}

Figure 2. Representative series of voltammograms recorded in PBS, pH 7.4, over the range of scan rates of interest at the CCF0 Carbostar conical carbon fiber electrode used in this study without (black $\mathrm{CV}$ traces) or with $30 \mu \mathrm{M}$ dopamine added (red $\mathrm{CV}$ traces). Potentials are referred to a $\mathrm{Ag} / \mathrm{AgCl} \mathrm{pseudo}$ reference electrode.

at CCF1-4 electrodes. In all cases, the voltammograms were background subtracted following the standard procedure introduced by Wightman and coll. ${ }^{38}$ to enhance the Faradic currents components (Figure 3).

Figure 4 discloses the scan rate dependence of the peak potential values for the forward and backward background-corrected waves before or after compensation of their ohmic drop shifts based on the following approximate correction:

$$
E_{i R-\text { corr }}^{p}(v)=E_{\text {back-corr }}^{p}(v)-i_{\text {tot }}^{p}(v) R
$$

where $R$ is the ohmic resistance determined from the initial slope of the background currents at $v=5 \mathrm{kV} . \mathrm{s}^{-1},{ }^{40}$ viz., $R=72 \mathrm{k} \Omega$; $E_{\text {back-corr }}^{p}(v)$ is the oxidation or reduction peak potential measured at a given scan rate $v$ from background-subtracted CVs; $i_{\mathrm{tot}}^{p}(v)$ is the maximum current intensity of the $\mathrm{CV}$ wave measured at the same scan rate before background subtraction. Note that $R$ was found to be ca. 2.5 higher than the value $(27 \pm 2 \mathrm{k} \Omega)$ determined by impedance upon assuming a simple $\mathrm{RC}$ equivalent circuit.

Figure 5a displays the variations with the scan rate of the background-corrected peak current intensities, $i_{\text {corr }}^{p}(v)$, of the forward voltammetric waves for conical electrodes CCF1-4.

\section{Discussion}

Analysis of the results.-All the following quantitative analyses were performed using background-corrected data generated at conical microelectrodes CCF1-4 (CCF0 electrode yielded background and faradaic currents that were ca. twice those from the batch CCF1-4 so they were discarded from the following quantitative analyses). First of all we must insist on the fact that although we resorted to the usual arithmetic subtraction method ${ }^{38}$ for background correction of the voltammograms in order to enhance Faradic contributions, Savéant et al. ${ }^{40}$ established that this method is not theoretically correct because it does not take into account the coupling between Faradic and capacitive components that occurs as soon as the Faradic current develops. However, we applied the usual subtraction method ${ }^{38}$ because (i) we wished to perform this study in line with the framework of usual practice of FSCV, and (ii) because the method proposed by Savéant and coll. ${ }^{40}$ can be applied with accuracy only when the capacitance is nearly independent of the potential, which is certainly not the case as evidenced by the background CVs (Figure 2). Nonetheless, since the current intensities of the subtracted CVs relative to the background ones do not vary excessively (i.e., at most by a factor ca. 2 over the range of scan rates investigated here, Figure 2), the bias introduced by the arithmetic background correction has to remain more or less constant over the whole series of CVs. In other words, even if the following analyses certainly have theoretical weaknesses in an absolute electrochemical sense, they are fully coherent with the usual practice in using FSCV for investigating dynamic fluctuations of catecholamines concentrations in the living brain. Nonetheless, these intrinsic limitations prevented developing and testing a more comprehensive quantitative kinetic analysis of the data than that given here.

Based on the square-scheme redox mechanism dominated by the adsorption/desorption processes established at classical carbon fiber disk electrodes, ${ }^{17-19}$ one would expect the current peak intensities of the background corrected CVs, $i_{\text {corr }}^{p}(v)$, to vary linearly with the scan rate over the range of scan rates considered in this study. However, the data reported in Figure 5a contradict such expectations. Indeed, $i_{\text {corr }}^{p}(v)$ varied linearly with the scan rate only in the low range of $v$ values (viz., $v<100 \mathrm{~V} . \mathrm{s}^{-1}$ ) but progressively drifted away from 

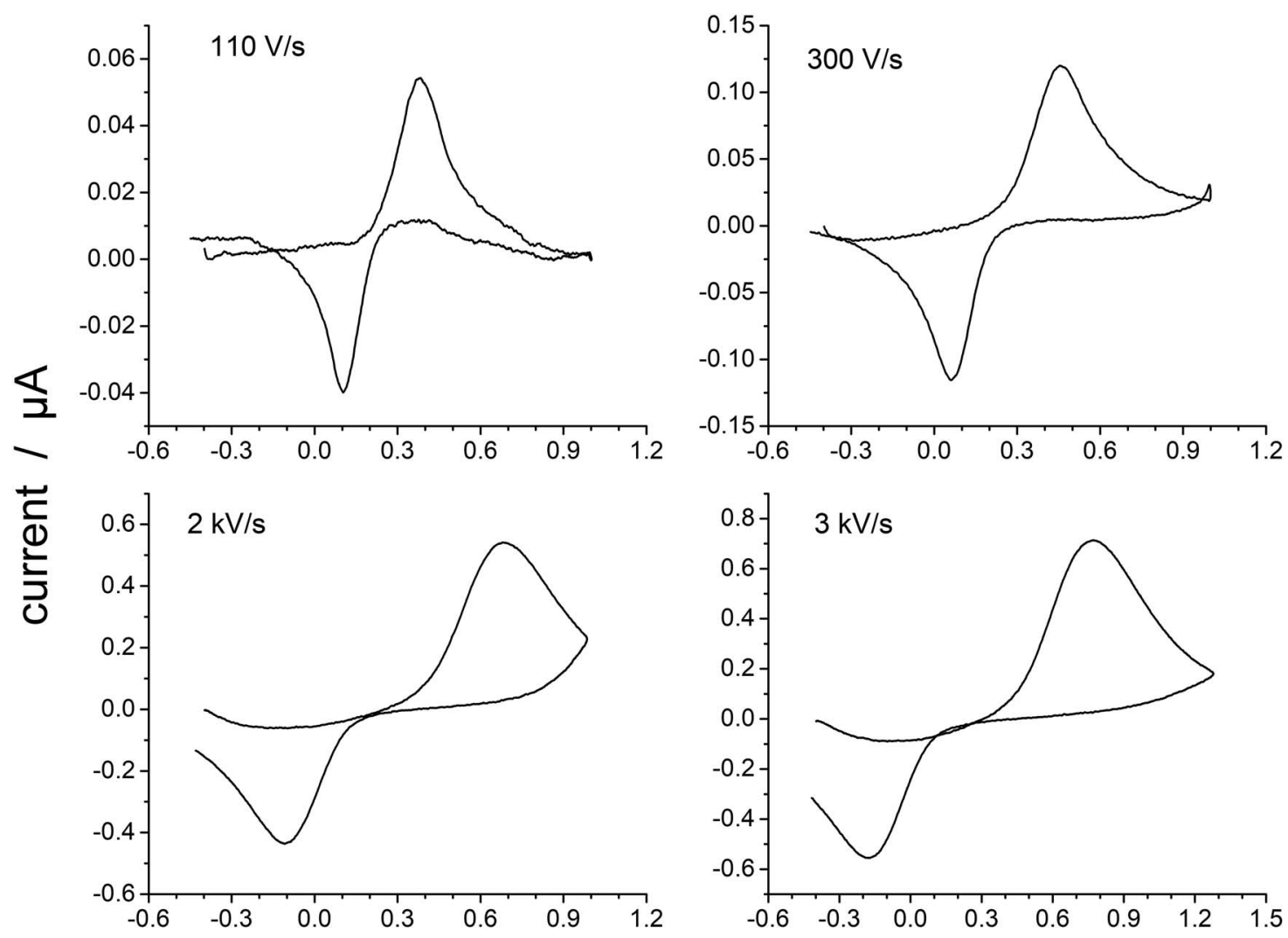

E / V vs. Ag/AgCl

Figure 3. Background-corrected voltammograms as extracted from the typical CV data presented for CCF0 electrode in Figure 2 for $30 \mu \mathrm{M}$ dopamine in PBS at $\mathrm{pH}$ 7.4. Potentials are referred to a $\mathrm{Ag} / \mathrm{AgCl}$ pseudo reference electrode.

linearity upon increasing $v$. It is noted that the slopes of the $i_{\text {corr }}^{p}(v)$ vs. $v$ variations in the lower $v$ range were extremely similar for all electrodes (Table I). A linear variation, i.e., $i_{\text {corr }}^{p} \propto v$, was recovered for electrode CCF1 in the upper range of $v$ values $\left(v>2 \mathrm{kV} \cdot \mathrm{s}^{-1}\right)$ though the corresponding slope $\left(0.11 \pm 0.01 \mathrm{nA} . \mathrm{s} . \mathrm{V}^{-1}\right)$ was smaller

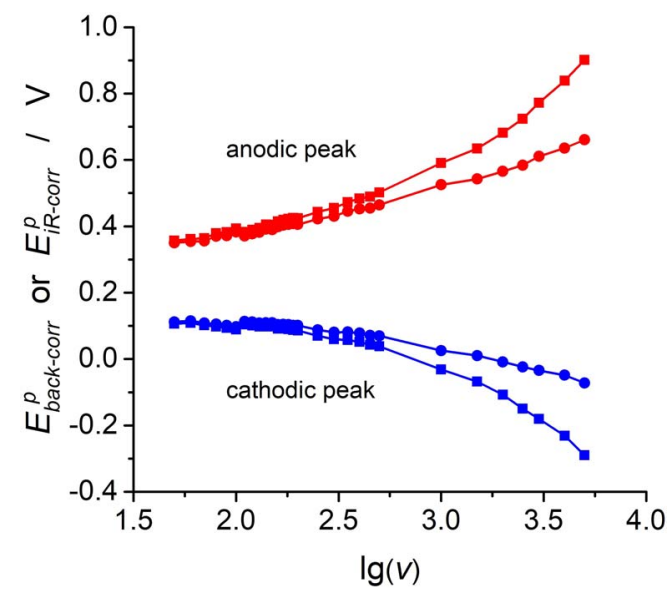

Figure 4. Variations of the forward (red color: anodic current peak) and backward (blue color: cathodic current peak) peak potential values recorded for $30 \mu \mathrm{M}$ dopamine in PBS at pH 7.4 at the CCF0 Carbostar conical electrode used for the voltammetric data shown in Figures 2 and 3. Squares: $E_{\text {back-corr }}^{p}(v)$ values; circles: $E_{i R-c o r r}^{p}(v)$ as obtained from Eq. 1 using $R=72 \mathrm{k} \Omega$ (see text and Experimental section). Peak potentials values are referred to a $\mathrm{Ag} / \mathrm{AgCl}$ pseudo reference electrode. than that observed in the lower scan rate range $\left(0.28 \pm 0.01 \mathrm{nA} . \mathrm{s} . \mathrm{V}^{-1}\right.$, Figure $5 \mathrm{~b}$ and Table I). The drift of $i_{\text {corr }}^{p}(v)$ vs. $v$ variations away from linearity when $v$ increased above $100 \mathrm{~V} . \mathrm{s}^{-1}$ (Figure 5a) could not result from any artefact due to the treatment of the data. Indeed, they were fully coherent with the gradual change of the shapes of the CV waves in Figure 3, from near bell-shaped $\mathrm{CV}^{39}$ at $v=110 \mathrm{~V} \cdot \mathrm{s}^{-1}$ to shapes reminiscent of diffusional control at larger scan rates.

This surprising behavior cannot be related to any mechanistic change of the redox rate-determining step because the $i R$ and background-corrected peak potentials (Figure 4) followed a monotonous linear variation with $\log (v)$ whose mean slope (ca. $165 \mathrm{mV}$ per unit of $\log (v))$ corresponded ${ }^{43,44}$ to a transfer coefficient $\alpha \sim 0.36$ for the rate-determining 1e-oxidation step of dopamine. ${ }^{19}$ Would the deviations from the linear $i_{\text {corr }}^{p} \propto v$ dependence feature a shift from an adsorption-controlled mechanism at $v<100 \mathrm{~V} . \mathrm{s}^{-1}$ to a control by diffusion ${ }^{42}$ from the solution one when the scan rate became too rapid vs. the adsorption rate, ${ }^{45-48}$ the transitions noticeable in the $i_{\text {corr }}^{p}(v)$ plots would be mirrored in the $E_{i R-c o r r}^{p}(v)$ plots. ${ }^{43}$ This was not the case experimentally (Figure 4). Moreover, assuming a local diffusional dependence, viz., that $i_{\exp }^{p}(v) \propto v^{1 / 2}$ over the range $v>100 \mathrm{~V} . \mathrm{s}^{-1}$, afforded an unrealistically large diffusion coefficient value for dopamine in a PBS solution. Hence, it is safely concluded that the oxidation process exclusively involved mechanisms occurring in adsorbed phase as was established at carbon disk microelectrodes. ${ }^{17,18}$

A second possible hypothesis which could have explained a drift of $i_{\text {corr }}^{p}(v)$ away from linearity in adsorbed films consisted in assuming that at high scan rates the oxidation process was limited by electron-hopping ${ }^{49-55}$ within a dopamine film (viz., $i_{\text {corr }}^{p}(v) \rightarrow v^{1 / 2}$ ) while the whole film could be oxidized at small scan rates (viz., $\left.i_{\text {corr }}^{p}(v) \propto v\right) .{ }^{56-59}$ This could apparently account for the drift of the $i_{\text {corr }}^{p}(v)$ functions away from linearity as observed in Figure 5a for 

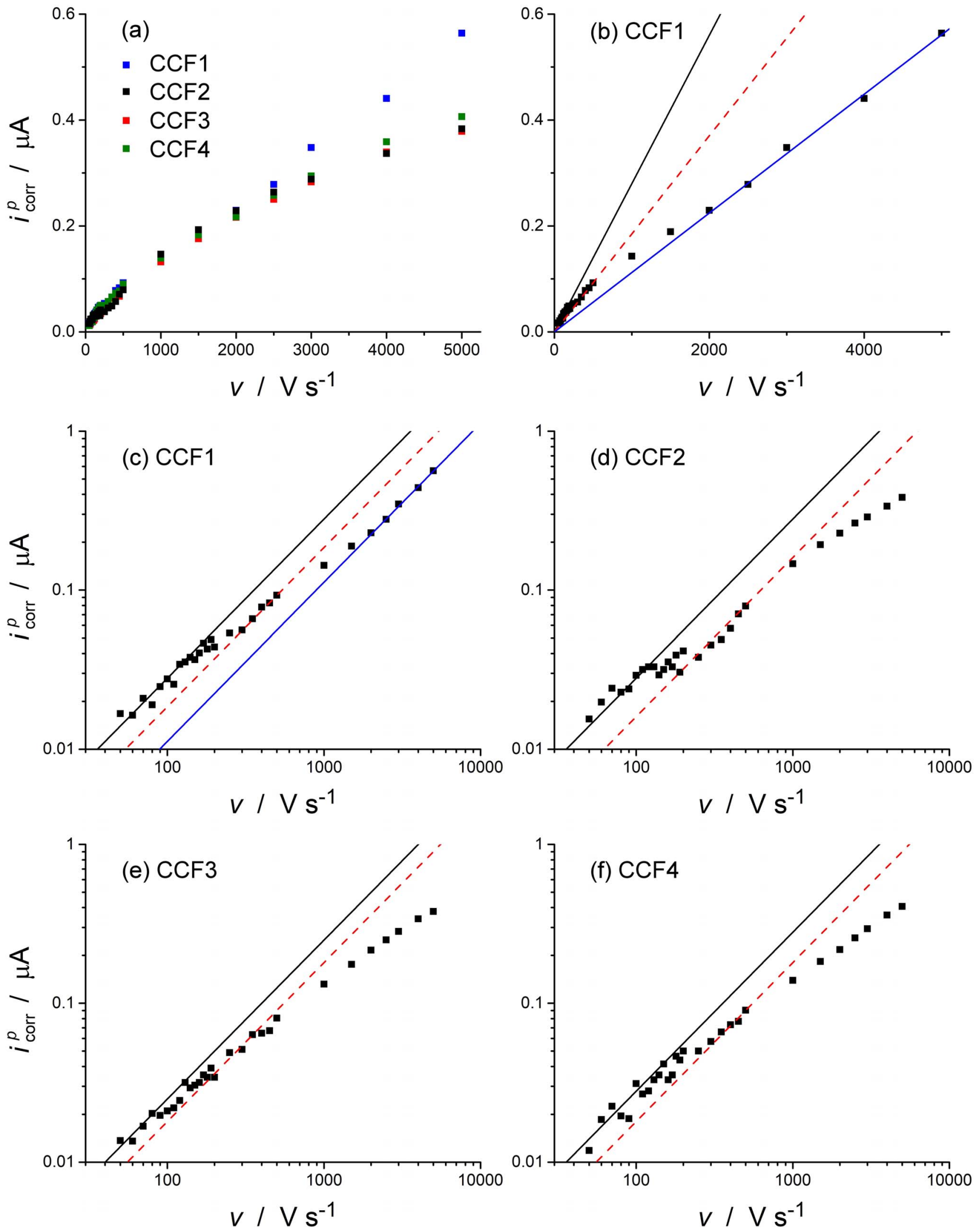

Figure 5. Variations of the oxidation wave peak current intensity, $i_{\text {corr }}^{p}(v)$, of background-corrected voltammograms as a function of the scan rate for each of the 4 Carbostar conical carbon fiber electrodes (CCF1-CCF4) used in this study. $30 \mu \mathrm{M}$ dopamine in PBS at pH 7.4. (a) Superimposition of current peak intensities variations for all CCF electrodes as indicated. (b,c) Individual data for carbon fiber electrode CCF1 plotted using normal coordinates (b) or a log-log representation (c). (d-f) Variations of the current peak intensities with the scan rate presented in log-log plots for the three other CCF electrodes. The regression lines shown in black correspond to the linear behaviors, $i_{\text {corr }}^{p} \propto v$, observed in the low scan rate range (ca. $v<100 \mathrm{~V} . \mathrm{s}^{-1}$; Table I). See text and Table I for the meaning of the other regression lines shown in (b-f). 


\section{Table I. Values of the slopes of the linear sections of the $i_{c o r r}^{p}(v)$ vs. $v$ variations as determined for each conical carbon fiber electrode CCF1-CCF4} from the log-log plots shown in Figures 5c-5f (see text).

\begin{tabular}{cccc} 
Electrode & $\begin{array}{c}\text { Slopes }\left(\mathrm{nA} . \mathrm{s} . \mathrm{V}^{-1}\right) \\
\text { Low scan rates range } \mathrm{a}^{\mathrm{a}} \\
\left(\text { ca. } v<100 \mathrm{~V} . \mathrm{s}^{-1}\right)\end{array}$ & $\begin{array}{c}\text { Intermediate scan range }^{\mathrm{b}} \\
\left(\text { ca. } 250<v<1000{\left.\mathrm{~V} . \mathrm{s}^{-1}\right)}^{-1}\right.\end{array}$ & $\begin{array}{c}\text { Large scan rates range }^{\mathrm{c}} \\
\left(\text { ca. } v>2000 \mathrm{~V} . \mathrm{s}^{-1}\right)\end{array}$ \\
\hline $\mathrm{CCF} 1$ & $0.28 \pm 0.01$ & $0.18 \pm 0.01$ & $0.11 \pm 0.01$ \\
$\mathrm{CCF} 2$ & $0.28 \pm 0.01$ & $0.16 \pm 0.01$ & $\mathrm{~N} / \mathrm{A}$ \\
$\mathrm{CCF} 3$ & $0.25 \pm 0.01$ & $0.18 \pm 0.01$ & $\mathrm{~N} / \mathrm{A}$ \\
$\mathrm{CCF} 4$ & $0.28 \pm 0.01$ & $0.18 \pm 0.01$ & $\mathrm{~N} / \mathrm{A}$ \\
mean & 0.27 & 0.175 & N/A
\end{tabular}

${ }^{a}$ shown as the solid black lines in Figures 5b-5f.

${ }^{\mathrm{b}}$ Shown as the dashed red lines in Figures $5 \mathrm{~b}-5 \mathrm{f}$.

${ }^{\mathrm{c}}$ Shown as the solid blue lines in Figures 5b, 5c.

electrodes CCF2-CCF4 but was in total contradiction with the behaviors of the $i_{\text {corr }}^{p}(v)$ functions above ca. $200-300 \mathrm{~V} . \mathrm{s}^{-1}$ for all electrodes (Figures 5c-5f). Hence, this hypothesis was also discarded.

Rationalization of the results.-Let us first focus on electrode CCF1 since it displayed a distinctive clear-cut behavior (Figures 5b, $5 c)$. Indeed, the log-log plot of $i_{\text {corr }}^{p}(v)$ vs. $v$ data in Figure $5 c$ for electrode CCF1 evidenced the presence of two asymptotic limits with unity slopes, viz., $i_{\text {corr }}^{p} \propto v$, reached either in the small scan rates range (ca., $v<100 \mathrm{~V} . \mathrm{s}^{-1}$ ) or the large one (ca., $v>2 \mathrm{kV} . \mathrm{s}^{-1}$ ), being connected by a transition at intermediate $v$ values. For this reason, we wish first to focus our analysis on the data determined for electrode CCF1.

In each extreme scan rate range the oxidation of dopamine at electrode CCF1 revealed a kinetic behavior coherent with a limiting process dominated by electrochemistry in adsorbed film. This suggests that at least two domains with different surface areas, $A_{l \text { or } h}$, and different dopamine surface concentrations, $\Gamma_{l}$ or $h$, where the subscripts $l$ or $h$ stand for 'low' or ' $h i g h$ ' scan rate, respectively, contribute sequentially as a function of time. Note that considering the co-existence of different types of nanodomains randomly distributed on the electrode surface is consistent with the internal structures of etched PAN carbon fibers (see Experimental section). Indeed, etching procedures are then expected to expose different types of carbon surfaces (compare the wide panel of surfaces morphologies evidenced in the SEM photographs shown in Figures 1b-1e) whose chemical and electronic structures, hence their electrochemical properties, must differ. ${ }^{31-34} \mathrm{Ev}$ idently, one cannot exclude that more than two types of nanodomains are involved. Yet, at this stage, a model restricted to two nanodomains is sufficient to delineate a theoretical ground on which more complex situations may be elaborated whenever required (see below).

Within this first approximation, the current peak variations in the low scan rate range may then be formulated as: ${ }^{39}$

$$
\left[i_{\text {corr }}^{p}(v)\right]_{v<100 \mathrm{V.s}^{-1}}=\Gamma_{l} A_{l} v \times\left[\alpha n F^{2} /(2.718 R T)\right]
$$

with $n=2^{19}$ and $\alpha=0.36$ as deduced from the $E_{i R-\operatorname{corr}}^{p}(v)$ vs. $\log v$ variations in Figure 4. Conversely, in the high scan rate one, one has:

$$
\left[i_{\text {corr }}^{p}(v)\right]_{v>2 \mathrm{kV} . \mathrm{s}^{-1}}=\Gamma_{h} A_{h} v \times\left[\alpha n F^{2} /(2.718 R T)\right]
$$

Note that in Eqs. 2 or 3 we use a factor $n$ rather than $n^{2}$ because the two electrons are not transferred simultaneously but sequentially through a ECEC square-scheme mechanism ${ }^{60}$ in a sequence of two independent single electron transfer steps, ${ }^{19}$ each one being followed by an acid-base reaction. ${ }^{61}$

Within this framework, only the small population of $\Gamma_{h} A_{h}$ adsorbed dopamine molecules may be electroactive at high scan rates, giving rise to the small slope of the linear variations of $i_{\text {corr }}^{p}(v)$ vs. $v$ (Figures 5b, 5c and Table I). Conversely, the larger slope observed at small scan rates (Figures $5 \mathrm{~b}, 5 \mathrm{c}$ and Table I) indicates that a larger population containing $\Gamma_{l} A_{l} \approx 2.5 \times \Gamma_{h} A_{h}$ adsorbed dopamine molecules was involved as can be inferred from the relative slopes at low and high scan rates in Figure 5b and Table I. Such a shift between populations

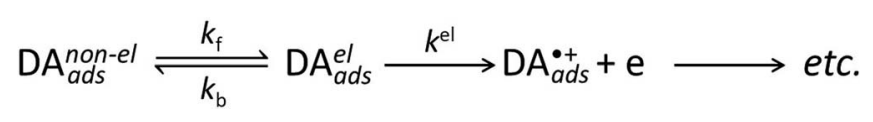

Scheme 1. Schematic mechanism describing the kinetic involvement of dopamine molecules initially adsorbed on one type of non-electroactive carbon sites.

cannot be ascribed to drastically different electron transfer rates or thermodynamic constants since this would contradict the absence of observable transitions in the $E_{i R-\text { corr }}^{p}(v)$ vs. $\log v$ variations ${ }^{39,43}$ (Figure 4). To the best of our knowledge, the only possibility that may account for such behavior consists in considering that the first oxidation step proceeded through the surface equivalent of a classical CE solution mechanism $^{62}$ as sketched in Scheme 1 . Within this mechanism, only a single population of dopamine molecules located on electroactive sites (noted $\mathrm{DA}_{a d s}^{e l}$ ) is responsible for the whole electrochemical response at all scan rates. However, depending on the scan rate, the progressive depletion of $\mathrm{DA}_{\text {ads }}^{e l}$ molecules by their oxidation may be compensated $\left(v<100 \mathrm{~V} \cdot \mathrm{s}^{-1}\right)$ or not $\left(v>1 \mathrm{kV} \cdot \mathrm{s}^{-1}\right)$ by the migration of another population of dopamine molecules (noted $\mathrm{DA}_{a d s}^{\text {non-el }}$ ) that were initially adsorbed onto non-electroactive domains.

At sufficiently small scan rates, the exchange between the two populations is fully dynamic, so the oxidation of the electroactive $\mathrm{DA}_{a d s}^{e l}$ molecules continuously displaces the $\mathrm{DA}_{a d s}^{n o n-e l} \rightleftharpoons \mathrm{DA}_{a d s}^{e l}$ equilibrium that regenerates them. At low scan rates, the whole set of adsorbed molecules may then be oxidized irrespective of the electrochemical activity of the domains on which they had adsorbed before the $\mathrm{CV}$ scan was performed. Then, the total quantity of oxidized molecules in this low scan rates range is $\Gamma_{l} A_{l}=(1+\gamma) \Gamma_{h} A_{h}$, so that:

$$
\left[i_{\text {corr }}^{p}(v)\right]_{v<100{\mathrm{~V} . \mathrm{s}^{-1}}}=(1+\gamma) \Gamma_{h} A_{h} v \times\left[\alpha n F^{2} /(2.718 R T)\right]
$$

with $\gamma=\left(\Gamma_{l} A_{l}-\Gamma_{h} A_{h}\right) / \Gamma_{h} A_{h} \approx 1.5$ for electrode CCF1. Conversely, when the scan rate gradually increased above $100 \mathrm{~V} \cdot \mathrm{s}^{-1}$ the $\mathrm{DA}_{a d s}^{\text {non-el }} \rightleftharpoons \mathrm{DA}_{a d s}^{e l}$ equilibrium displacement became less and less efficient up to the point when it resulted totally frozen $\left(v>2 \mathrm{kV} \cdot \mathrm{s}^{-1}\right.$ for electrode CCF1). From this point, only the $\mathrm{DA}_{a d s}^{e l}$ dopamine molecules that were initially adsorbed onto electroactive nanopatches of the carbon surface may be oxidized during one voltammetric scan, hence:

$$
\left[i_{\text {corr }}^{p}(v)\right]_{v>2 \mathrm{kV} . \mathrm{s}^{-1}}=\Gamma_{h} A_{h} v \times\left[\alpha n F^{2} /(2.718 R T)\right]
$$

Would the ratio $k_{\mathrm{b}} / k_{\mathrm{f}}$ in Scheme 1 had a significant value one should observe a significant shift in the $E_{i R-\operatorname{corr}}^{p}(v)$ vs. $\log v$ variations reflecting the change in global thermodynamics occurring in phase with the transition between the two current limits. Indeed, the thermodynamic oxidation potential of $\mathrm{DA}_{a d s}^{\text {non-el }}$ would then be higher than that of $\mathrm{DA}_{a d s}^{e l}$ by $(R T / F) \ln \left(1+k_{\mathrm{b}} / k_{\mathrm{f}}\right),{ }^{62}$ and possibly result in the development of a second oxidation wave. ${ }^{21}$ Figures 3 and 4 do not provide any evidence for such behavior so it is inferred that 
$k_{\mathrm{b}} \ll k_{\mathrm{f}}$. In other words, when a fresh CCF1 electrode is dipped into the solution, dopamine adsorbs most favorably onto the electroactive nanodomains compared to the non-electroactive ones. This is rather consistent with the fact that adsorption of catecholamines onto carbon materials is generally assumed to involve supramolecular binding to oxidized carbons moieties, viz., is facilitated at the edges or defects of basal planes, i.e., at areas where electron transfers seem also to be facilitated. ${ }^{21,29-32}$

The time course required to reach the current peak during one voltammogram is approximately $\theta \sim R T / F v .^{63}$ Thus, during this time duration $\theta$ the depletion of the initial $\mathrm{DA}_{a d s}^{e l}$ molecules may be compensated by the progressive migration and subsequent oxidation of a quantity $q(\theta)=\gamma \Gamma^{*} A^{*} \times f(\theta)$ of $\mathrm{DA}_{a d s}^{n o n-e l}$ molecules $(f(\theta)$ is an appropriate kinetic term characteristic of the kinetically-controlled migration process taking place between the randomly dispersed nanodomains structures). In the absence of other information on the nature of the surface migration kinetics, and assuming that the nanodomains of different types are numerous and distributed statistically over the electrode surface (compare Figures 1b-1e), we limit ourselves to the simplest kinetic equation that may apply under such circumstances. This amounts to assume that the diffusional equivalent of the Newton and Kelvin law of cooling applies, i.e., that diffusional equilibration within each type of nanodomains is much faster than exchanges between adjacent ones. ${ }^{64,65}$ This leads at each instant to the fast setting of a quasi-steady state inside each nanodomain, so that $f(\theta)=\left[1-\exp \left(-k_{\mathrm{f}} \theta\right)\right] \cdot{ }^{64,65}$ It follows that to describe the current peak intensity at a given scan rate one may consider that the electrochemical system behaves as if it initially consisted of a single domain containing $\Gamma_{h} A_{h}\left\{1+\gamma\left[1-\exp \left(-k_{\mathrm{f}} R T / F v\right)\right]\right\}$ oxidizable adsorbed molecules irrespective of the fact that only $\Gamma_{h} A_{h}$ of them were initially adsorbed onto electroactive nanopatches before the voltammetric scan was initiated. In practice the kinetics of $\mathrm{DA}_{a d s}^{n o n-e l}$ molecules migration may be more intricate, e.g., involving radial or cylindrical diffusion according to the exact nanodomain shapes and their imbrication onto the electrode surface, ${ }^{41,66,67}$ thus leading to more complex formulations of $f(\theta)$. Yet, in the absence of specific information, we decided to limit ourselves to the above quasi-steady state approximation in the following.

Interestingly, one should note that while a $\mathrm{CV}$ wave is monitored beyond its current peak the time scale $\theta$ keeps increasing and may reach values equal to several times $R T / F v$ along the $\mathrm{CV}$ descending branch. Therefore, unless it was already almost complete at the level of the CV peak, the amount of $\mathrm{DA}_{a d s}^{n o n-e l}$ molecules that may migrate and be oxidized onto the electroactive areas progressively increases while the descending branch of the voltammetric peak is displayed. Such progressive displacement of the $\mathrm{DA}_{a d s}^{n o n-e l} \rightleftharpoons \mathrm{DA}_{a d s}^{e l}$ equilibrium along the CVs descending branches perfectly justifies the fact that over the range of scan rates featuring the transition between the two limiting linear behaviors $\left(100 \mathrm{~V} . \mathrm{s}^{-1}<v<2 \mathrm{kV} . \mathrm{s}^{-1}\right.$, Figure 3$)$ the $\mathrm{CV}$ waves displayed features reminiscent of diffusional processes and not the classical bell-shapes expected for processes undergoing in adsorbed phase. ${ }^{39}$

Finally, it is interesting to contrast this mechanism in regards of the ca. 2.5 factor found between the solution resistances determined through impedance assuming a simple RC circuit and that determined theoretically as noted in the Experimental section. Indeed, a random distribution of nanodomains of different electroactivities at the electrode surface cannot be considered as leading to a classical RC formulation. This would require a good homogeneity of the surface electroactivity. Conversely, a random distribution of nanodomains implies a collection of RC elements acting in parallel and whose global outcome cannot be described by that of a single RC circuit. ${ }^{68,69}$ Furthermore, the nanometric curvatures of the electric field toward the electroactive domains impose local constrictions of the current tubes so that the global solution resistance results drastically larger ${ }^{68}$ than would be predicted for a totally active conical electrode. Though this fact cannot be taken as a proof of the nano-heterogeneity of the $\mathrm{CCF}$ surface electroactivity, its coherence with our assumption is worth mentioning.

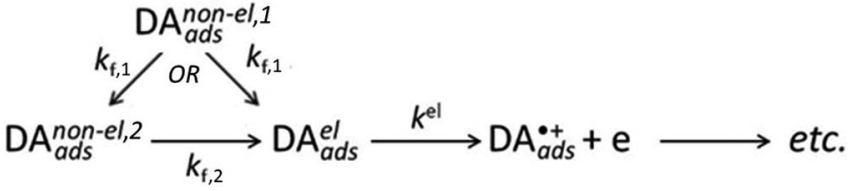

Scheme 2. Schematic mechanisms describing the kinetic involvement of dopamine molecules initially adsorbed on two types of non-electroactive carbon sites.

Let us now discuss the behavior of electrode CCF2 based on the rationale developed for CCF1. Indeed, not only this behavior does not differ from that of electrode CCF1 as significantly as it may appear, but its analysis will help refine the simple two-nanodomain model elaborated for rationalizing the data relative to electrode $\mathrm{CCF} 1$. The transition between the two domains occurs sooner and over a shorter range (ca. $90 \mathrm{~V} . \mathrm{s}^{-1}<v<200 \mathrm{~V} . \mathrm{s}^{-1}$, Figure 5d) for CCF2 than CCF1 while reaching a more important limiting value of the $i_{\text {corr }}^{p}(v)$ vs. $v$ slope in the high scan rate range (viz., $0.16 \pm 0.01 \mathrm{nA} . \mathrm{s} . \mathrm{V}^{-1}$ vs. $0.11 \pm 0.01 \mathrm{nA} . \mathrm{s} . \mathrm{V}^{-1}$, compare Table I). The only drastic difference in behavior between the two electrodes is thus related to the fact that for CCF $2 i_{\text {corr }}^{p}(v)$ undergoes a second non-linear transition above $1 \mathrm{kV} . \mathrm{s}^{-1}$ while this was not the case for CCF1 (compare Figures $5 \mathrm{c}$ and $5 \mathrm{~d}$ ).

These results allow implementing further our initial rationalization of the behavior of electrode CCF1. Indeed, one may consider that the rather long transition between the two linear limits observed in Figure $5 \mathrm{c}$ features a sequence of two transitions involving an intermediate linear stage delineated by the dashed red line in Figures $5 \mathrm{~b}, 5 \mathrm{c}$ whose slope $\left(0.18 \pm 0.01 \mathrm{nA} . \mathrm{s} . \mathrm{V}^{-1}\right)$ is almost identical to that observed for electrode $\mathrm{CCF} 2$ over the same scan rate range $\left(0.16 \pm 0.01 \mathrm{nA} . \mathrm{s} . \mathrm{V}^{-1}\right)$. This can be easily accounted within the above mechanistic framework through considering the co-existence of two populations, $\mathrm{DA}_{a d s}^{n o n-e l, 1}$ and $\mathrm{DA}_{a d s}^{\text {non-el,2 }}$, containing respectively $\gamma_{1} \Gamma_{h} A_{h}$ and $\gamma_{2} \Gamma_{h} A_{h}$ dopamine molecules initially adsorbed onto two non-electroactive domains, both prone to regenerate the oxidized $\mathrm{DA}_{a d s}^{e l}$ molecules within different time scales imposed by their corresponding rate constants $k_{\mathrm{f}, 1}$ and $k_{\mathrm{f}, 2}$ with $k_{\mathrm{f}, 1} \ll k_{\mathrm{f}, 2}$. This involves two parallel or sequential CE sequences regenerating $\mathrm{DA}_{a d s}^{e l}$ molecules similar to the single one sketched in Scheme 1 (see Scheme 2). Hence, during one voltammogram, the whole system behaves as if it would initially consist of a single domain containing $\Gamma_{h} A_{h}\left\{1+\gamma_{1}\left[1-\exp \left(-k_{\mathrm{f}, 1} R T / F v\right)\right]+\gamma_{2}\left[1-\exp \left(-k_{\mathrm{f}, 2} R T / F v\right)\right]\right\}$ oxidizable dopamine molecules. Then, one expects to observe two transitions separating the three $i_{\text {corr }}^{p}(v)$ vs. $v$ linear regimes:

$$
i_{\text {corr }}^{p}(v)=\left(1+\gamma_{1}+\gamma_{2}\right) \Gamma_{h} A_{h} v \times\left[\alpha n F^{2} /(2.718 R T)\right]
$$

within the smallest scan rate range (i.e., $v<100 \mathrm{~V} . \mathrm{s}^{-1}$ for electrode CCF1), followed by:

$$
i_{\text {corr }}^{p}(v)=\left(1+\gamma_{2}\right) \Gamma_{h} A_{h} v \times\left[\alpha n F^{2} /(2.718 R T)\right]
$$

in the intermediate scan rate range (i.e., $200 \mathrm{~V} . \mathrm{s}^{-1}<v<700 \mathrm{~V} . \mathrm{s}^{-1}$, for electrode CCF1), and ultimately by:

$$
i_{\text {corr }}^{p}(v)=\Gamma_{h} A_{h} v \times\left[\alpha n F^{2} /(2.718 R T)\right]
$$

at higher scan rates (i.e., $v>2 \mathrm{kV} . \mathrm{s}^{-1}$, for electrode CCF1). From the relative values of the slopes of $i_{\text {corr }}^{p}(v)$ vs $v$ variations in Table I, one deduces that $\gamma_{1} \approx 0.9$ and $\gamma_{2} \approx 0.6$ for electrode CCF1.

This mechanistic framework unifies the behaviors observed for electrodes CCF1 and CCF2 showing that their apparent differences stem only from slight differences of their respective $\gamma_{1}, \gamma_{2}, k_{\mathrm{f}, 1}$ and $k_{\mathrm{f}, 2}$ parameters. Interestingly, the total quantity of adsorbed molecules, viz., the $\left(1+\gamma_{1}+\gamma_{2}\right) \Gamma_{h} A_{h}$ values are similar for all four electrodes as evidenced by the almost common initial slopes of the $i_{\text {corr }}^{p}(v)$ vs. $v$ variations at $v<100 \mathrm{~V}$. $\mathrm{s}^{-1}$ (Figure 5, Table I). In fact, the only drastic difference between CCF1 and CCF2-4 electrodes is that the third asymptotic behavior in Eq. 8 is not observable for electrodes CCF2-4, presumably, because it occurs at scan rates larger than those investigated here. 
One may imagine that in a general situation the two/threenanodomain model elaborated above can be extended by assuming the co-existence of a single type of electroactive nanodomains containing $\Gamma_{h} A_{h}$ adsorbed dopamine molecules $\mathrm{DA}_{a d s}^{e l}$ interwoven with several non-electroactive ones, each one containing a quantity $\gamma_{j} \Gamma_{h} A_{h}$ of initially adsorbed dopamine molecules, $\mathrm{DA}_{a d s}^{n o n-e l, j}$. Still assuming a quasi-steady state kinetic control of the migration of $\mathrm{DA}_{a d s}^{n o n-e l, j}$ molecules to replace the oxidized $\mathrm{DA}_{a d s}^{e l}$ ones, this predicts a general formulation of the current peak intensity $i_{\text {corr }}^{p}(v)$ dependence with the scan rate given as in Eq. 9:

$$
\begin{aligned}
i_{\text {corr }}^{p}(v) & =\left[\alpha n F^{2} \Gamma_{h} A_{h} v /(2.718 R T)\right] \\
& \times\left\{1+\sum \gamma_{j}\left[1-\exp \left(-k_{\mathrm{f}, j} R T / F v\right)\right]\right\}
\end{aligned}
$$

Interestingly, Eq. 9 predicts that all conical electrodes of same global surface area must behave extremely similarly at small scan rates. This is coherent with the fact that the four CCF conical electrodes investigated here displayed $i_{\text {corr }}^{p}(v)$ vs. $v$ linear variations with almost identical slopes in this time range (Table I; solid black lines in Figures $5 \mathrm{~b}-5 \mathrm{f})$. This reflects that in this range $\left(v<100 \mathrm{~V} . \mathrm{s}^{-1}\right)$ the dynamic interchanges between all nanodomains are fast enough to allow all adsorbed dopamine molecules to be oxidized via $\mathrm{CE}$ sequences such as those in Schemes 1 and 2 irrespective of the electroactivity of the nanodomain on which they initially adsorbed. Conversely, the exact nature of the nanodomain surface areas and the surface dynamics of the $\mathrm{DA}_{a d s}^{n o n-e l, j}$ molecules, hence the specific values of each set of $\gamma_{j}$ and $k_{\mathrm{f}, j}$ parameters pertaining to one given electrode, matter more and more at larger scan rates.

\section{Conclusions}

This work has evidenced that even if the presence of nanoscale heterogeneities of electroactive domains at carbon electrodes surface has been known since the earliest use of carbon fiber electrodes for measurement of catecholamines fluxes and concentration in brain, ${ }^{28,29}$ they have important dynamic consequences on voltammetric measurements that have been ignored up to now (at least to the best of our knowledge). However, these surface dynamic processes strongly modulate the scan rate dependent relationships between concentrations and current intensities on the in FSCV. In particular, the general assumption that at fast scan rates current intensities scale linearly with the scan rate may not be always correct.

This seems to justify the common practice of pre- and postcalibrating high aspect ratio carbon fiber electrodes using FSCV conditions identical to those used during measurements in the brain. ${ }^{36}$ However, the relationship between concentrations and current intensities not only depends on the surface states and their distribution but also on the time scale of all phenomena involved in each media (brain or solution). During pre- or post-calibrations in solutions as was done in this work the access of the dopamine to the electrode surface is even, so the only time scales to consider are those of the FSCV waveform function. ${ }^{36}$ However, when the electrode is inserted in a brain area where the distribution of releasing sites is not homogeneous ${ }^{37}$ one needs to envision the possible effect of another time scale related to the diffusion time of released molecules toward the carbon fiber electrode. ${ }^{14}$ Indeed, owing to the fast dynamics evidenced in this work, molecules initially adsorbed on nanopatches closest to the release sites may migrate rather fast to cover sections of the electrode located rather far away, thus allowing the whole surface of the electrode to be loaded with neurotransmitter and not only the nanodomains that are in close contact with the cluster(s) of releasing sites. Though, as sketched in Figure 6, this necessarily imposes a subtle scan rate regulated interplay between the rates featuring the different diffusional paths involved in bringing the neurotransmitter at the carbon fiber electrode surface and the ensuing migration steps between the different sites distributed on it. Hence, depending on the exact placement of a given carbon fiber electrode in a non-homogeneous region of the brain, this kinetic interplay may lead to discrepancies vis-à-vis what
A) Pre- or Post-Calibration

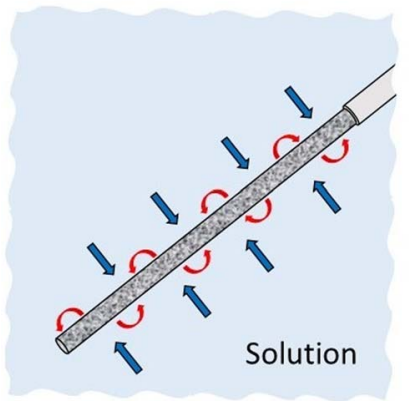

B) In-vivo Measurement

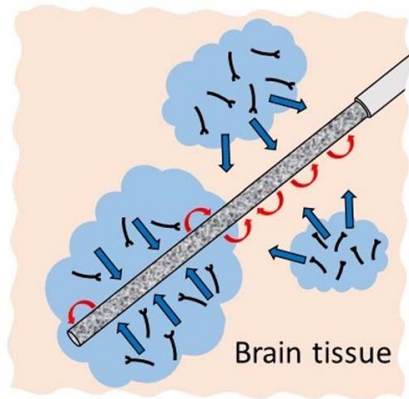

Figure 6. Schematic representation of transport phenomena involving a neurotransmitter detected at a large aspect-ratio carbon fiber electrode during preor post-calibration ${ }^{36}$ in a solution of the neurotransmitter (A) or during an in-vivo measurement (B) when the neurotransmitter is released within clusters distributed in the probed area. ${ }^{37}$ In $(A, B)$ transport to the electrode surface is represented by dark blue arrows while migration between non-electroactive and electroactive nanodomains intermingled on the carbon fiber surface is represented by red curved arrows. In (B) the blue-colored domains figure the release-active clusters containing a high synapses density while the surrounding less active brain tissue is indicated in light color. The texture of the carbon fiber electrode symbolizes the interwoven nanodomains of different electroactivity on which the neurotransmitter adsorbs.

may be inferred from pre- and post-calibrations. To the best of our knowledge, this complex kinetic dimension has never been considered before this work.

\section{Acknowledgments}

This work was performed in collaboration between the groups from Paris, France (ENS, UMR8640) and Aarhus, Denmark (iNANO). In Paris, it was supported in parts by CNRS UMR 8640, ENS (Ecole Normale Superieure), PSL and Sorbonne as well as by LIA CNRS NanoBioCatEchem. In Aarhus, it was supported by the EU, the H2020-MSCA-IF-2014 grant agreement 660339 (eADAM). CA also acknowledges Xiamen University for a Distinguished Professor position. The authors gratefully acknowledge the Denes Budai/Kation Scientific Company for the permission of using some of the SEM microphotographs of Carbostar conical carbon fiber microelectrodes published on their web site and adapting them in composing Figure 1.

\section{ORCID}

Alexander Oleinick (1D https://orcid.org/0000-0001-6194-0459

Irina Svir (D) https://orcid.org/0000-0002-6006-3148

Elena E. Ferapontova (iD https://orcid.org/0000-0003-1177-3204

Christian Amatore (D) https://orcid.org/0000-0002-2561-1137

\section{References}

1. See: (a) G. Grant, in "The Nobel Prizes in the field of neuroscience", http://www.nobelprizemedicine.org/selecting-laureates/history /the-nobel-prizes-in-the-field-of-neuroscience/

2. Liljestrand, The Nobel Prize in Physiology or Medicine 1932, cited in Nobel Lectures, Physiology or Medicine 1922-1941, Elsevier Publishing Company, Amsterdam, 1965.

3. E. G. Gray and R. W. Guillery, Int. Rev. Cytol., 19, 111 (1966)

4. R. N. Adams, Prog. Neurobiol., 35, 297 (1990)

5. R. M. Wightman, L. J. May, and A. C. Michael, Anal. Chem., 60, 769A (1988).

6. D. L. Robinson, A. Hermans, A. T. Seipel, and R. M. Wightman, Chem. Rev., 108, 2554 (2008).

7. C. Amatore, S. Arbault, M. Guille, and F. Lemaître, Chem. Rev, 108, 2585 (2008)

8. L. Ren, L. Mellander, J. Keighron, A-S. Cans, M. Kurczy, I. Svir, A. Oleinick, C. Amatore, and A. G. Ewing, Quarter. Rev. Biophys., 49, e12 (2016).

9. W.-Z. Wu, W.-H. Huang, W. Wang, Z.-L. Wang, J.-K. Cheng, T. Xu, R.-Y. Zhang, Y. Chen, and J. Liu, J. Am. Chem. Soc., 127, 8914 (2005)

10. Y. T. Li, S. H. Zhang, L. Wang, R. R. Xiao, W. Liu, X. W. Zhang, Z. Zhou, C. Amatore, and W. H. Huang, Angew. Chem. Int. Ed., 53, 12456 (2014). 
11. Y. T. Li, S. H. Zhang, X. Y. Wang, X. W. Zhang, A. I. Oleinick, I. Svir, C. Amatore and W. H. Huang, Angew. Chem. Int. Ed., 54, 9313 (2015).

12. S. Majdi, E. C. Berglund, J. Dunevall, A. I. Oleinick, C. Amatore, D. Krantz, and A. G. Ewing, Angew. Chem. Int. Ed., 7, 13609 (2015).

13. J. E. Baur, E. W. Kristensen, L. J. May, D. J. Wiedemann, and R. M. Wightman, Anal Chem., 60, 1268 (1988).

14. C. Amatore, R. S. Kelly, E. W. Kristensen, W. G. Kuhr, and R. M. Wightman, J. Electroanal. Chem., 213, 31 (1986).

15. S. H. Walters, E. M. Robbins, and A. C. Michael, ACS Chem. Neurosci., 6, 1468 (2015)

16. A. M. Andrews, ACS Chem. Neurosci., 8, 211 (2017)

17. M. K. Zachek, A. Hermans, R. M. Wightman, and G. S. McCarty, J. Electroanal. Chem., 614, 113 (2008).

18. B. D. Bath, D. J. Michael, B. J. Trafton, J. D. Joseph, P. L. Runnels, and R. M. Wightman, Anal. Chem., 72, 5994 (2000).

19. E. L. Ciolkowski, K. M. Maness, P. S. Cahill, R. M. Wightman, D. H. Evans, B. Fosset, and C. Amatore, Anal. Chem., 66, 3611 (1994).

20. C. Amatore, S. Arbault, Y. Bouret, M. Guille, F. Lemaitre, and Y. Verchier, Anal. Chem., 81, 3087 (2009).

21. I. Alvarez-Martos and E. E. Ferapontova, Electrochem. Commun., 89, 48 (2018).

22. L. A. Sombers, L. R. Wilson, C. F. Mason, and C. A. Lee, Abstract 2303, 232nd ECS Meeting, National Harbor, USA (2017).

23. Rapid Dopamine Release in Freely Moving Rats. D. L. Robinson and R. M. Wightman, in Electrochemical Methods for Neuroscience, A. C. Michael and L. M. Borland, Editors, Chapter 2, CRC Press/Taylor \& Francis, Boca Raton (2007).

24. J. J. Clark, S. G. Sandberg, M. J. Wanat, J. O. Gan, E. A. Horne, A. S. Hart, C. A. Akers, J. G. Parker, I. Willuhn, V. Martinez, S. B. Evans, N. Stella, and P. E. M. Phillips, Nat. Methods, 7,126 (2010).

25. C. Dugast, R. Cespuglio, and M. F. Suaud-Chagny, J. Neurochem., 82, 529 (2002).

26. J. Park, B. J. Aragona, B. M. Kile, R. M. Carelli, and R. M. Wightman, Neuroscience, 169, 132 (2010).

27. D. Budai, I. Hernadi, B. Meszaros, Z. K. Bali, and K. Gulya, Acta Biologica Szegediensis, 54, 155 (2010).

28. J.-L. Ponchon, R. Cespuglio, F. Gonon, M. Jouvet, and J.-F. Pujol, Anal. Chem., 51, 1483 (1979)

29. F. Gonon, M. Buda, R. Cespuglio, M. Jouvet, and J.-F. Pujol, Nature, 286, 902 (1980).

30. K. T. Kawagoe, J. B. Zimmerman, and R. M. Wightman, J. Neurosci. Methods, 48 , 225 (1993).

31. R. L. McCreery, K. K. Cline, C. A. McDermott, and M. T. McDermott, Colloid Surface A, 93, 211 (1994).

32. P. Chen, M. A. Fryling, and R. L. McCreery, Anal. Chem., 67, 3115 (1995)

33. C. H. Lin, L. F. Chen, E. E. L. Tanner, and R. G. Compton, Chem. Sci., 9, 152 (2018)

34. T. J. Davies, M. E. Hyde, and R. G. Compton, Angew. Chem. Int. Ed., 44, 5121 (2005).

35. A. N. Patel, S. Y. Tan, T. S. Miller, J. V. Macpherson, and P. R. Unwin, Anal. Chem., 85, 11755 (2013)

36. M. J. Logman, E. A. Budygin, R. R. Gainetdinov, and R. M. Wightman, J. Neuroscience Meth., 95, 95 (2000).

37. D. L. Robinson, B. J. Venton, M. L. A. V. Heien, and R. M. Wightman, Clinical Chem., 10, 1763 (2003).
38. J. O. Howell, W. G. Kuhr, R. E. Ensman, and R. M. Wightman, J. Electroanal. Chem., 209, 77 (1986).

39. A. J. Bard and L. R. Faulkner, Electrochemical methods. Fundamentals and applications, 2nd ed., Chap. 14, p. 594, Wiley \& Sons, New York (2001).

40. L. Nadjo, J. M. Savéant, and D. Tessier, J. Electroanal. Chem., 52, 403 (1974).

41. C. Amatore, J.-M. Savéant, and D. Tessier, J. Electroanal. Chem., 147, 39 (1983).

42. C. Amatore, in Physical Electrochemistry: Principles, Methods and Applications, I. Rubinstein, Editor, Chap.4. pp. 131, M. Dekker, New York (1995).

43. L. Nadjo and J. M. Savéant, J. Electroanal. Chem., 48, 113 (1973).

44. A. J. Bard and L. R. Faulkner, Electrochemical methods. Fundamentals and applications, 2nd ed., Chap. 6, p. 231 \& 236, Wiley \& Sons, New York, USA (2001).

45. Y. F. Huang, D. Y. Wu, A. Wang, B. Ren, S. Rondinini, Z. Q. Tian, and C. Amatore, J. Am. Chem. Soc., 132, 17199 (2010).

46. O. V. Klymenko, I. Svir, and C. Amatore, J. Electroanal. Chem., 688, 320 (2013).

47. O. Klymenko, O. Buriez, E. Labbe, D. P. Zhan, S. Rondinini, Z. Q. Tian, and C. Amatore, ChemElectroChem, 1, 227 (2014).

48. O. V. Klymenko, I. Svir, and C. Amatore, Molecular Physics, 112, 1273 (2014).

49. H. J. Dahms, J. Phys. Chem., 72, 362 (1968).

50. I. Ruff and V. J. Friedrich, J. Phys. Chem., 75, 3297 (1971).

51. I. Ruff, V. J. Friedrich, K. Demeter, and K. Csillag, J. Phys. Chem., 75, 3303 (1971).

52. C. P. Andrieux and J. M. Savéant, J. Electroanal. Chem., 111, 377 (1980)

53. R. W. Murray, Phil. Trans. R. Soc. London A., 302, 253 (1981).

54. E. Laviron, J. Electroanal. Chem., 112, 1 (1980).

55. D. N. Blauch and J. M. Savéant, J. Am. Chem. Soc., 114, 3323 (1992).

56. C. Amatore, Y. Bouret, E. Maisonhaute, J. I. Goldsmith, and H. D. Abruna, ChemPhysChem, 2, 130 (2001).

57. C. Amatore, Y. Bouret, E. Maisonhaute, J. I. Goldsmith, and H. D. Abruna, Chem. Eur. J., 7, 2206 (2001).

58. P. Fortgang, E. Maisonhaute, C. Amatore, B. Delavaux-Nicot, J. Iehl, and J.-F. Nierengarten, Angew. Chem. Int. Ed., 50, 2364 (2011).

59. X.-S. Zhou, B.-W. Mao, C. Amatore, R. G. Compton, J.-L. Marignier, M. Mostafavi, J.-F. Nierengarten, and E. Maisonhaute, Chem. Commun., 52, 251 (2016).

60. E. Laviron and L. Roullier, J. Electroanal. Chem., 186, 1 (1985).

61. See a discussion of this point in the context of solution processes in: C. Amatore, M. Azzabi, P. Calas, A. Jutand, C. Lefrou, and Y. Rollin, J. Electroanal. Chem., 288, 45 (1990).

62. J. M. Savéant and E. Vianello, Electrochim. Acta, 8, 905 (1963).

63. C. Amatore, in Organic Electrochemistry, 5th ed., O. Hammerich and B. Speiser, Editors, Table 5, p. 79, CRC Press, Boca Raton (2016).

64. A. Oleinick, R. Hu, B. Ren, Z. Q. Tian, I. Svir, and C. Amatore, J. Electrochem. Soc., 163, H3014 (2016).

65. A. Oleinick, I. Svir, and C. Amatore, Proc. Royal Soc. A, 473, 20160684 (2017).

66. O. Sliusarenko, A. Oleinick, I. Svir, and C. Amatore, Electroanalysis, 27, 980 (2015).

67. O. Sliusarenko, A. Oleinick, I. Svir, and C. Amatore, ChemElectroChem, 2, 1279 (2015).

68. See e.g., C. Amatore, M. Berthou, and S. Hébert, J. Electroanal. Chem., 457, 191 (1998).

69. C. Amatore, A. Oleinick, O. V. Klymenko, and I. Svir, Anal. Chem., 81, 8545 (2009). 